\title{
The complex relation between production and scattering amplitudes
}

\author{
Eef van Beveren \\ Centro de Fúsica Teórica, Departamento de Fúsica, Universidade de Coimbra \\ P-3004-516 Coimbra, Portugal \\ http://cft.fis.uc.pt/eef \\ George Rupp \\ Centro de Física das Interacções Fundamentais, Instituto Superior Técnico \\ Universidade Técnica de Lisboa, Edifício Ciência, P-1049-001 Lisboa, Portugal \\ george@ist.utl.pt \\ PACS number(s): 11.80.Gw, 11.55.Ds, 13.75.Lb, 12.39.Pn
}

August 22, 2021

\begin{abstract}
The unitarity relation $\Im m(A)=T^{*} A$ is derived for a three-body production amplitude $A$ that consists of a complex linear combination of elements of the two-body scattering amplitude $T$. We conclude that the unitarity relation does not impose a realness condition on the coefficients in the expansion of $A$ in terms of $T$.
\end{abstract}

Under the spectator assumption, we deduced in Ref. [1] that the three-particle production amplitude $A$ consists of a complex linear combination of elastic and inelastic matrix elements of the two-body scattering amplitude $T$. Furthermore, in Ref. [2] we showed that such a twoparticle production amplitude can reasonably describe experiment in an energy region where no additional resonances from possible rescattering with the spectator particle exist. Moreover, no need to treat a sizable fraction of the experimental signal as background was noticed.

The result of Ref. [1] agrees to some extent with the expression proposed in Refs. [3,4]. Like in our Ref. [1], the authors of Ref. [4] based their ansatz on the OZI rule [5] and the spectator picture, finding that the production amplitude can be written as a linear combination of the elastic and inelastic two-body scattering amplitudes, with coefficients that do not carry any singularities, but are rather supposed to depend smoothly on the total CM energy of the system.

However, Ref. [4] concluded from the unitarity relation

$$
\Im m(A)=T^{*} A
$$

that the production amplitude must be given by a real linear combination of the elements of the transition matrix. A similar conclusion, based on a $K$-matrix parametrisation, can be found in 
Ref. [6]. In contrast, we arrive at a different conclusion, namely that the coefficients must be complex, in agreement with experiment [7-9] as well as with the work of the Ishidas [10,11].

Relation (11), which can also be found in Ref. [12], basically stems from the operator relations $A V=(1+T G) V=V+T G V=T$, the symmetry of $T$, the realness of $V$, and the unitarity of $1+2 i T$, which gives $\Im m(A) V=\Im m(A V)=\Im m(T)=T^{*} T=T^{*} A V$. This leads, for non-singular potentials $V$, to relation (11).

Now we shall show that $A$ and $T$ satisfy the unitarity relation (1) despite the complexness of the coefficients in the expansion of $A$ in terms of $T$. Thereto, we are going to strip the expressions of Ref. [1] of all details which might obscure the simplicity of our arguments. Hence, let $Z_{k}(E)(k=1,2, \ldots, n)$ represent a vector of complex non-singular expressions 1 , being smooth functions of the energy $E$, where $n$ represents the number of coupled scattering channels under consideration, and let the relation between $A$ and $T$ be given by 2

$$
A_{k}=\Re e\left(Z_{k}\right)+i \sum_{\ell} Z_{\ell} T_{k \ell}
$$

We then find for the imaginary part of the production amplitude

$$
\Im m\left(A_{k}\right)=\frac{1}{2 i}\left(A_{k}-A_{k}^{*}\right)=\sum_{\ell}\left\{\Re e\left(Z_{\ell}\right) \Re e\left(T_{k \ell}\right)-\Im m\left(Z_{\ell}\right) \Im m\left(T_{k \ell}\right)\right\} .
$$

Next, we substitute on the right-hand side of Eq. (3) the identity $\Re e\left(T_{k \ell}\right)=T_{k \ell}^{*}+i \Im m\left(T_{k \ell}\right)$, and furthermore insert the unitarity condition for $T$, i.e., $\Im m\left(T_{k \ell}\right)=\sum_{\ell^{\prime}} T_{\ell^{\prime} \ell} T_{k \ell^{\prime}}^{*}$, so as to obtain

$$
\begin{aligned}
\Im m\left(A_{k}\right) & =\sum_{\ell}\left\{\Re e\left(Z_{\ell}\right)\left(T_{k \ell}^{*}+i \Im m\left(T_{k \ell}\right)\right)-\Im m\left(Z_{\ell}\right) \Im m\left(T_{k \ell}\right)\right\} \\
& =\sum_{\ell}\left\{\Re e\left(Z_{\ell}\right) T_{k \ell}^{*}+i Z_{\ell} \sum_{\ell^{\prime}} T_{\ell^{\prime} \ell} T_{k \ell^{\prime}}^{*}\right\} .
\end{aligned}
$$

Finally, we interchange $\ell$ and $\ell^{\prime}$ in the second term on the right-hand side of Eq. (4), leaving us, also using Eq. (2), with

$$
\Im m\left(A_{k}\right)=\sum_{\ell} T_{k \ell}^{*}\left\{\Re e\left(Z_{\ell}\right)+i \sum_{\ell^{\prime}} Z_{\ell^{\prime}} T_{\ell \ell^{\prime}}\right\}=\sum_{\ell} T_{k \ell}^{*} A_{\ell} .
$$

This completes the proof that A, as defined in Eq. (2), satisfies the unitarity condition (11). Consequently, relation (1) does not impose a realness condition on the coefficients in Eq. (2).

The first term on the right-hand side of relation (2) was not considered in Refs. [3, 4, 6]. However, in the works of Graves-Morris [13] and Aitchison \& collaborators [14-16], the possible existence of an additional real contribution was anticipated. In Refs. [1,2], this follows straightforwardly from the reasonable assumption that a produced meson pair originates from an initial $q \bar{q}$ pair. As a consequence, the observed phenomenological necessity [9] to employ complex coefficients in experimental analyses of production processes does not allow by itself to draw conclusions on the inevitability of including rescattering diagrams with the spectator particle in theoretical approaches.

\footnotetext{
${ }^{1}$ In Appendix $\mathrm{A}$ we give the precise relation between the expressions used in Ref. [1] and $Z_{k}$.

${ }^{2}$ Note that for $\Re e\left(Z_{k}\right)=0$ one obtains an expansion with real coefficients, as in Refs. [3,4].
} 


\section{Acknowledgements}

We wish to thank I. J. R. Aitchison, D. V. Bugg, C. Hanhart and M. R. Pennington for useful discussions. This work was supported in part by the Fundação para a Ciência e a Tecnologia of the Ministério da Ciência, Tecnologia e Ensino Superior of Portugal, under contract PDCT/ $\mathrm{FP} / 63907 / 2005$.

\section{A Precise definition of $Z_{k}(E)$}

In Ref. [1] we discussed the partial-wave expansion of the amplitudes for two-meson production - together with a spectator particle - and scattering, assuming $q \bar{q}$ pair creation. Hence, the coefficients bear reference to the partial wave $\ell$ and the flavor content $\alpha$ of the quark pair. We obtained [1] the following relation between production and scattering partial-wave amplitudes:

$$
A_{\alpha i}^{(\ell)}=g_{\alpha i} j_{\ell}\left(p_{i} r_{0}\right) \sqrt{\mu_{i} p_{i}}+i \sum_{\nu} g_{\alpha \nu} \sqrt{\mu_{\nu} p_{\nu}} h_{\ell}^{(1)}\left(p_{\nu} r_{0}\right) T_{i \nu}^{(\ell)}
$$

Accordingly, we must define

$$
Z_{\alpha k}^{(\ell)}(E)=g_{\alpha k} h_{\ell}^{(1)}\left(p_{k} r_{0}\right) \sqrt{\mu_{k} p_{k}}
$$

In the latter equations, $j_{\ell}$ and $h_{\ell}^{(1)}$ stand for the spherical Bessel function and Hankel function of the first kind, respectively. These are smooth functions of the total CM energy, just like $\mu_{k}$ and $p_{k}$, which are the reduced mass and relative linear momentum of the two-meson system in the $k$-th channel, respectively. The constants $g_{\alpha k}$ stand for the intensities of the $q \bar{q} \rightarrow M M$ couplings. A distance scale $\sim 0.6 \mathrm{fm}$ (for light quarks) is represented by $r_{0}$. In the text we have stripped $Z$ of a reference to $\ell$ and $\alpha$.

Note, moreover, as can be easily seen from expressions (2) and (6), that the singularity structures of the production and scattering amplitudes are identical, since $\Re e\left(Z_{k}\right)$, which is proportional to the spherical Bessel function in Eq. (6), is a smooth function of the total invariant mass.

\section{References}

[1] E. van Beveren and G. Rupp, Relating multichannel scattering and production amplitudes in a microscopic OZI-based model, arXiv:0706.4119.

[2] E. van Beveren and G. Rupp, S-wave and P-wave pion-pion and kaon-pion contributions to three-body decay processes in the Resonance-Spectrum Expansion, J. Phys. G 34, 1789 (2007) arXiv:hep-ph/0703286.

[3] S. U. Chung, J. Brose, R. Hackmann, E. Klempt, S. Spanier and C. Strassburger, Partial wave analysis in $K$ matrix formalism, Annalen Phys. 507, 404 (1995).

[4] K. L. Au, D. Morgan and M. R. Pennington, Meson dynamics beyond the quark model: A study of final-state interactions, Phys. Rev. D 35, 1633 (1987). 
[5] S. Okubo, $\Phi$ meson and unitary symmetry model, Phys. Lett. 5, 165 (1963);

G. Zweig, An $\mathrm{SU}_{3}$ model for strong interaction symmetry and its breaking, CERN Reports TH-401 and TH-412 (1963);

see also Developments in the Quark Theory of Hadrons, Vol. 1, pp. 22-101 (1981), edited by D. B. Lichtenberg and S. P. Rosen;

J. Iizuka, K. Okada and O. Shito, Systematics and phenomenology of boson mass levels (3), Prog. Theor. Phys. 35, 1061 (1966).

[6] M. Boglione and M. R. Pennington, Towards a model independent determination of the $\phi \rightarrow f_{0} \gamma$ coupling, Eur. Phys. J. C 30, 503 (2003) arXiv:hep-ph/0303200.

[7] J. M. Link et al. [FOCUS Collaboration], Dalitz plot analysis of $D_{s}^{+}$and $D^{+}$decay to $\pi^{+} \pi^{-} \pi^{+}$ using the K-matrix formalism, Phys. Lett. B 585, 200 (2004) arXiv:hep-ex/0312040.

[8] P. Dini [FOCUS Collaboration], Dalitz plot analyses from FOCUS, Int. J. Mod. Phys. A 20, $482(2005)$.

[9] J. M. Link et al. [FOCUS Collaboration] and M. R. Pennington, Dalitz plot analysis of the $D^{+} \rightarrow K^{-} \pi^{+} \pi^{+}$decay in the FOCUS experiment, Phys. Lett. B 653, 1 (2007) arXiv:0705.2248 [hep-ex]].

[10] M. Ishida, S. Ishida and T. Ishida, Relation between scattering and production amplitudes: Concerning $\sigma$ particle in $\pi \pi$ system, Prog. Theor. Phys. 99, 1031 (1998) arXiv:hep-ph/9805319.

[11] M. Ishida, The present status on sigma and kappa meson properties: Relation between scattering and production amplitudes, AIP Conf. Proc. 688, 18 (2004).

[12] U. G. Meißner and J. A. Oller, $J / \psi \rightarrow \phi \pi \pi$ ( $K$ anti-K) decays, chiral dynamics and OZI violation, Nucl. Phys. A 679, 671 (2001) arXiv:hep-ph/0005253.

[13] P. R. Graves-Morris, S-matrix theory of final-state interactions, Nuovo Cim. A 50, 681 (1967).

[14] M. G. Bowler, M. A. V. Game, I. J. R. Aitchison and J. B. Dainton, Diffraction dissociation, the Deck mechanism and diffractive resonance production, Nucl. Phys. B 97, 227 (1975).

[15] I. J. R. Aitchison and M. G. Bowler, Rescattering effects in the Deck model, J. Phys. G 3, 1503 (1977).

[16] I. J. R. Aitchison, Are there important unitarity corrections to the isobar model?, Phys. Lett. B 84, 349 (1979). 